\title{
SCREENING FOR MELAS MUTATIONS IN YOUNG PATIENTS WITH STROKE OF UNDETERMINED ORIGIN
}

\author{
Adriana Bastos Conforto', Fabio luji Yamamoto, \\ Sueli Mieko Oba-Shinjo', Julio Guy C. Pinto², Maurício Hoshino², \\ Milberto Scaff', Suely Kazue Nagahashi Marie ${ }^{1}$
}

\begin{abstract}
Purpose: It has been suggested that mitochondrial disease may be responsible for a substantial proportion of strokes of indetermined origin. We have preliminarily screened for MELAS (mitochondrial encephalomyopathy, lactic acidosis, and stroke-like episodes) mutations in young patients with cryptogenic strokes. Method: The mitochondrial mutations A3243G and T3271C were investigated in 38 subjects aged less than 46 years. Group 1: 15 patients with cryptogenic strokes; Group 2: 3 patients with diagnosis of MELAS syndrome, including stroke-like episodes; Group 3: 20 healthy subjects. Results: The A3243G mutation was absent in all subjects in Groups 1 and 3 but was present in all subjects in Group 2. Conclusion: Our results do not support screening for these mutations to diagnose oligosymptomatic forms of MELAS in cryptogenic strokes in the absence of other features of the syndrome. We suggest that clinical findings should guide mitochondrial genetic testing.
\end{abstract}

KEY WORDS: cerebrovascular disease, diagnostic methods, mitochondrial disease, myopathy, cryptogenic stroke.

\begin{abstract}
Mutações relacionadas à síndrome de MELAS em pacientes jovens com acidente vascular cerebral de etiologia indeterminada

RESUMO - Propósito: Foi sugerido que mitocondriopatias possam ser responsáveis por uma proporção substancial de acidentes vasculares cerebrais de etiologia indeterminada. Realizamos um estudo preliminar de pesquisa de mutações relacionadas à síndrome de MELAS (encefalomiopatia mitocondrial, acidose lática e episódios "stroke-like") em pacientes jovens com acidentes vasculares cerebrais criptogênicos. Método: As mutações mitocondriais A3243G e T3271C em 38 indivíduos com menos de 46 anos. Grupo 1: 15 pacientes com acidentes vasculares cerebrais criptogênicos; Grupo 2: 3 pacientes com diagnóstico de síndrome de MELAS, incluindo episódios "stroke-like"; Grupo 3: 20 voluntários saudáveis. Resultados: A mutação A3243G esteve ausente em todos os indivíduos dos Grupos 1 e 3 mas esteve presente em todos os indivíduos do Grupo 2. Conclusão: Nossos resultados sugerem que não há utilidade em pesquisar estas mutações para diagnosticar formas oligossintomáticas de MELAS em acidentes vasculares cerebrais criptogênicos na ausência de características da síndrome. Sugerimos que o quadro clínico deva guiar a solicitação de pesquisas de mutações relacionadas a mitocondriopatias nestes pacientes .
\end{abstract}

PALAVRAS-CHAVE: doença cerebrovascular, métodos diagnósticos, mitocondriopatias, miopatias, infarto criptogênico.

Worldwide, cryptogenic strokes account for 8.3$55 \%$ of ischemic strokes in young patients ${ }^{1-5}$. It has been suggested that mitochondrial disorders may present oligosymptomatically and may be responsible for up to one third of cryptogenic ischemic strokes in the young ${ }^{6}$. Even though clinical features vary widely in mitochondrial diseases, distinctive syndromes such as MELAS (mitochondrial encephalomyopathy, lactic acidosis and stroke-like episodes) have been described $^{7-9}$. Stroke-like episodes can appear at any age in MELAS and occur in around $50 \%$ of the patients with the A3243G mitochondrial DNA (mtDNA) muta- tion $^{10}$. In most cases, episodes occur before the fifth decade. The occipital lobe is the most commonly affected area but the reason for this predilection is unclear ${ }^{11}$. The A3243G mutation has been reported in approximately $80 \%$ of cases of MELAS and other mtDNA mutations such as T3271C have also been described $^{12}$. The degree of heteroplasmia (the proportion of normal and mutant mitochondrial DNA in each tissue) is an important factor influencing variability of phenotypical presentation.

If the full-blown syndrome does not develop but mitochondrial dysfunction is present and contrib-

Neurology Division, Hospital das Clínicas/São Paulo University, São Paulo SP, Brazil (HC/FMUSP): ${ }^{1} M D$, PhD; ${ }^{2} M D$. Este projeto foi financiado pela FAPESP através do Auxílio 97/3802-6.

Received 25 October 2006, received in final form 16 January 2007. Accepted 17 February 2007.

Dra. Adriana Bastos Conforto - Departamento de Neurologia / HCIFMUSP - Avenida Dr. Enéas de Carvalho Aguiar $255 / 5084$ 05403-000 São Paulo SP - Brasil.E-mail: abconf@yahoo.com e abconf@usp.br 
utes to a sufficient number of cryptogenic strokes in young patients, then screening for MELAS mutations may allow for diagnosis in these patients. In order to investigate this issue, we have preliminarily investigated the relative frequency of A3243G and T3271C mtDNA mutations in young subjects with stroke of undetermined etiology, patients with MELAS and healthy volunteers.

\section{METHOD}

We studied 38 subjects aged 45 years or less, according to the following criteria:

Group 1 (14 patients) - Ischemic stroke of undetermined origin according to Trial of Org 10172 in Acute Stroke Treatment (TOAST) criteria ${ }^{13}$. CT and MRI was performed in all patients: in the acute phase in 10 patients, in the chronic phase (after 6 months) in 6 patients and in both phases in 6 patients. Neuroimaging confirmed lesions that were classified as anterior and/or posterior territory infarcts $^{14,15}$. Clinical features of MELAS were evaluated. Patients with incomplete evaluation of stroke etiologies were excluded. All patients underwent extensive clinical and neurological evaluation, electrocardiogram, hemogram, blood biochemistry, lipid profile, erythrocyte sedimentation rate, Chagas' disease serology, syphilis serology, coagulogram, protein S, protein C, antithrombin III, haemoglobin electrophoresis, antinuclear antibodies, rheumatoid factor, complement, lupus anticoagulant, anticardiolipin antibodies, duplex ultrasonography of extracranial vessels, echocardiogram (transesophageal, 14; transthoracic, 1). In addition, intracranial and extracranial arteries were evaluated in all patients, either by magnetic resonance angiography (13), digital subtraction angiography (9) or transcranial Doppler (9). Holter monitoring was performed in 7 patients. Evaluation was performed retrospectively between November, 2001 and June, 2002 after diagnosis of ischemic stroke, either during admission in the Neurology ward (8 patients) or during follow-up in the Stroke clinic (6 patients).

Group 2 (3 patients) - Patients that had been admitted to the Neurology clinic or ward with a diagnosis of MELAS and stroke-like episodes according to the following criteria: a) presence of at least one episode with clinical and neuroimaging (CT or MRI) features compatible with strokelike episode, i.e., acute onset of focal neurologic deficit and neuroimaging findings not always corresponding to a vascular distribution ${ }^{7,11}$; b) increase in lactate level in blood and/or CSF; c) morphological findings suggestive of a mitochondrial disorder in muscle biopsy. Clinical, imaging and laboratory information were retrospectively reviewed. Histological and histochemical analyses were performed according to the technique standardized by Dubowitz ${ }^{16}$. The presence of ragged red fibers (RRFs) and the distribution of succinate desidrogenase positive $(\mathrm{SDH}+)$ fibers and $c y-$ tochrome $\mathrm{C}$ oxidase negative (COX-) fibers were investigated. The SDH reactivity of vessels was also evaluated. Increased number of mitochondria and paracrystalline inclusions were evaluated by electronic microscopy. Diffusion- weighted (DWI) MRI images were not available at the time the stroke-like episodes were diagnosed.

Group 3 (20 healthy subjects) - Inclusion criteria: Age and gender similar to patients in group 1. Exclusion criteria: neurological abnormalities (except history of headache). DNA analysis was performed in blood.

History of migraine according to criteria of the International Headache Society ${ }^{17}$, history of seizures, encephalopathy or recurrent vomiting were investigated in the three groups. In addition, the A3243G and T3271C mtDNA mutations were studied by PCR amplification of genomic DNA extracted from peripheral leukocytes by a salting out method ${ }^{18}$ and utilized for polymerase chain reaction (PCR)-restriction fragment length polymorphism. After endonuclease digestion specific for each point mutation (Apal for A3243G and AfIII for T3271C), products were analyzed by $8 \%$ polyacrilamide gel electrophoresis, followed by silver staining. Point mutational analysis was performed on digestion products.

In Group 2, the A3243G mutation was investigated in genomic DNA extracted from peripheral leukocytes and muscle biopsy specimens. In one patient, postmortem analysis was performed in the deltoid, diaphragm, extrinsic ocular and heart muscles as well as in the hypophysis, thyroid, liver and brain.

The research protocol followed institutional guidelines and was approved by the Ethics Committee of Hospital das Clínicas/ São Paulo University. Subjects gave informed consent.

MELAS is an extremely rare disease and, therefore, the number of patients in Group 2 was small. Therefore, we provided description of the data and did not perform formal statistical analysis.

\section{RESULTS}

The A3243G mutation was absent in all subjects in Groups 1 and 3 but was present in all patients in Group 2. The T3271C mutation was not found in any of the groups. Characteristics of subjects in Groups 1, 2 and 3 are shown in the Table.

Migraine headaches were present in 3/14 of subjects in Group 1, 4/14 of the subjects in Group 3 and 2/3 patients in Group 2. One patient in Group 1 had diabetes mellitus. No other symptoms of MELAS were present in Groups 1 or 3. Family history of stroke was present in 5/14 patients in Group 1. In Group 1, infarcts affected the posterior circulation territory in one third of the patients while in Group 2, all lesions included both anterior and posterior circulation territories but did not obey to strict limits of arterial territories (Fig 1).

Clinical and muscle biopsy findings typical of MELAS were present in all subjects in Group 2. In addition, maternal inheritance was present in all patients. Figure 2 shows the pedigree of Patient 3 as well as results of PCR amplification products. 

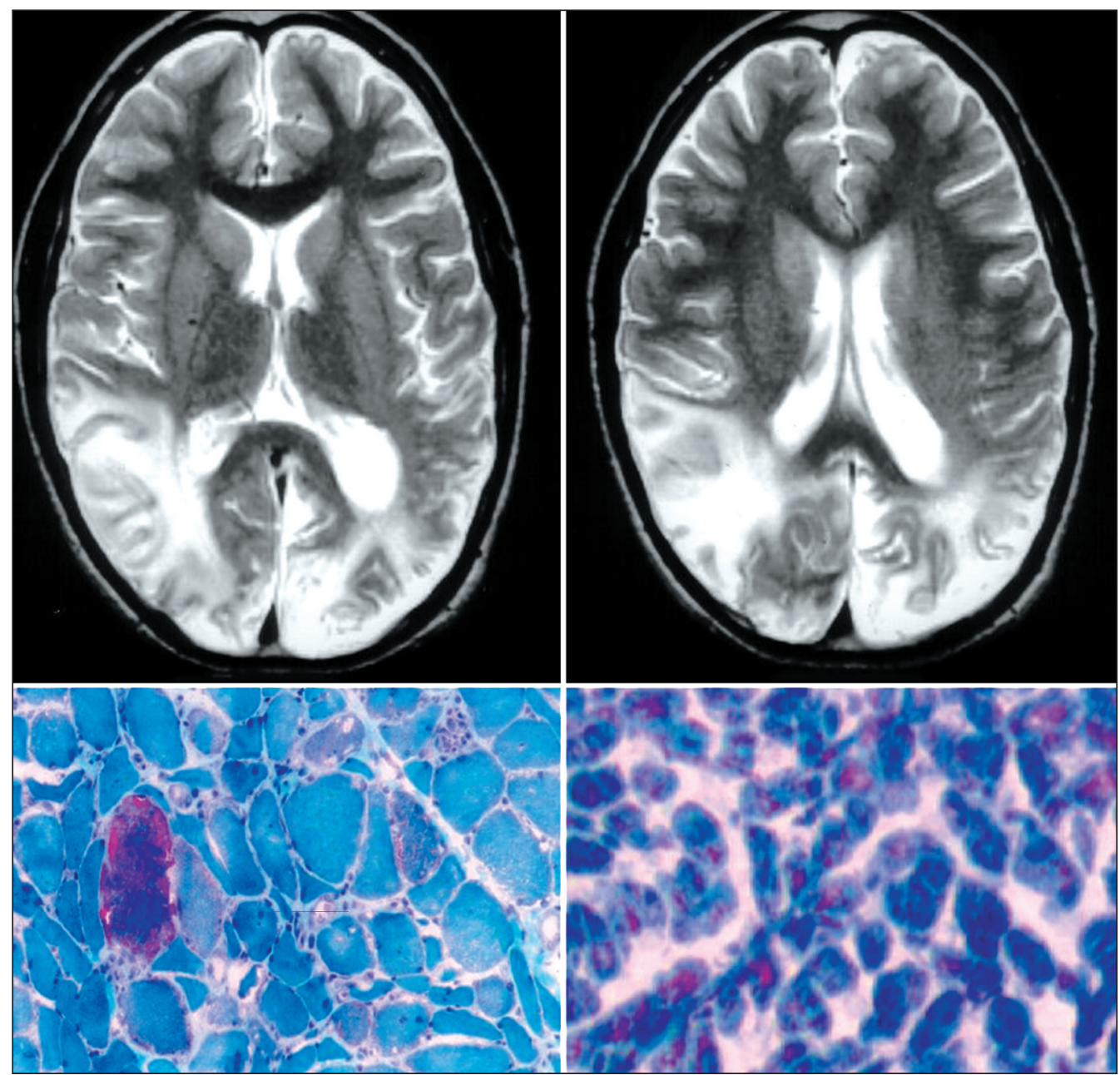

Fig 1. Top. T2-weighted MRI from Patient 3 (Group 2) shows increased signal in temporal cortex, occipital cortex and white matter bilaterally. Bottom. Postmortem findings in the same patient. Ragged red fibers (arrows) are present in diaphragm muscle (left) and heart muscle(right) stained by modified Gomori.

Patient 1 was a 32-year-old male with a history of seizures for the past five years. He also had episodes of encephalopathy and vomiting for the past four months prior to admission. He had no family history of similar symptoms. Lactate was increased in blood and CSF. MRI showed a stroke-like lesion involving the right temporal, parietal and occipital lobes as well as the right thalamus. The A3243G mutation was identified in DNA extracted from blood and muscle biopsy. Biopsy showed RRFs, SDH + and COX - fibers on light microscopy and an increased number of mitochondria on electron microscopy.

Patient 2 was female, presented with hearing loss at the age of 15 and migraine headaches at the age of 18. At age 29, she developed epilepsy and had a stroke-like episode. She had no family history of similar symptoms. MRI showed a right temporoparietal cortical lesion with increased signal in T2-weighted images. CT showed calcifications in globus pallidus and pulvinar bilaterally. Lactate was slightly in- creased in blood and CSF. Muscle biopsy showed RRF and SDH+ fibers. At the age of 34, she developed diabetes mellitus.

Patient 3 was male, began to have epileptic seizures at the age of 9 and one year later developed poor seizure control and sudden onset of right hemiparesis. He progressed with other spells of focal neurological deficits with partial resolution of the symptoms over a period of months. He had no family history of similar symptoms. His pedigree is shown in Figure 2. Lactate was increased in blood and CSF. Brain MRI is shown in Figure 1. Muscle biopsy showed RRFs, SDH+ and COX-fibers. Blood vessels from the muscle biopsy were also strongly $\mathrm{SDH}+$. He died at the age of 15 . Autopsy showed mitochondrial proliferation with RRFs on optic microscopy of deltoid, diaphragm, extrinsic ocular and heart muscles (Fig 1). Mitochondrial proliferation was also present in hypophysis, thyroid and liver. The brain showed cortical necrosis with vascular proliferation, calcium de- 


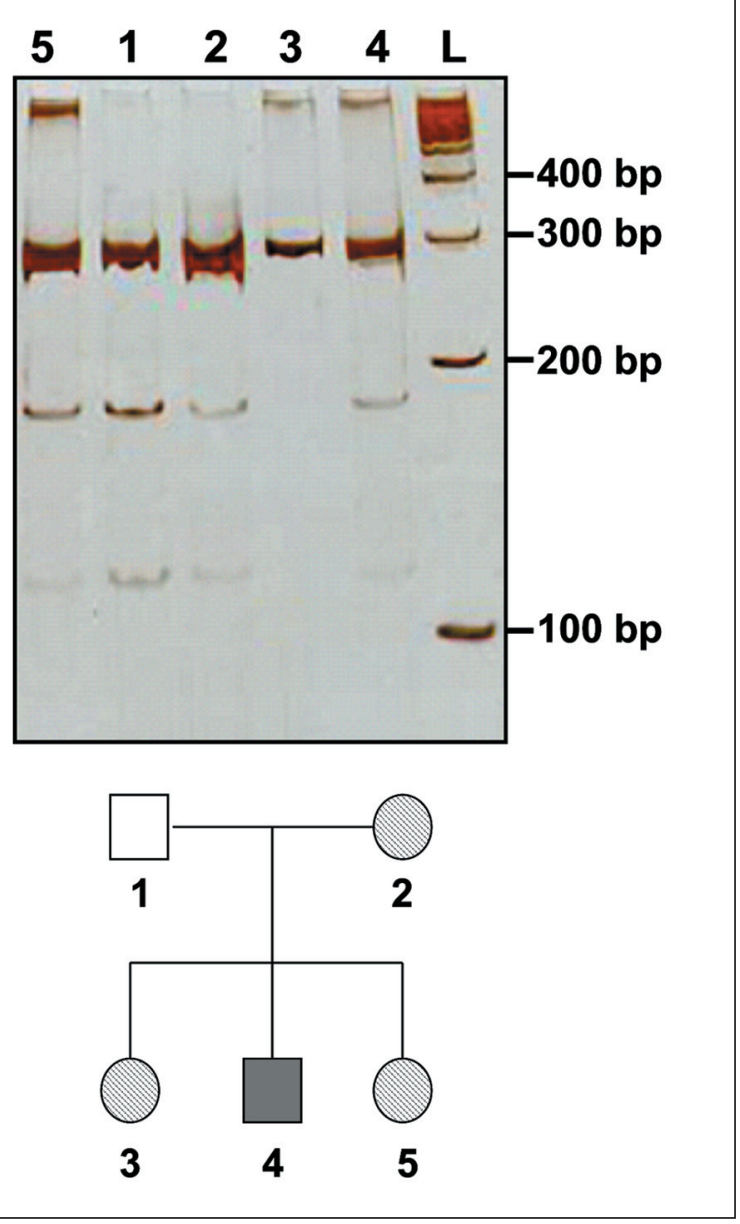

Fig 2. Pedigree from Patient 3 (bottom) and products of $P C R$ amplification digested with restriction enzyme Apal (top). The symptomatic patient (filled square) and the asymptomatic female carriers (circles) of A3243G mutation present two fragments (177 and $117 \mathrm{bp}$ ) in addition to the original $294 \mathrm{bp}$ fragment. Maternal inheritance is evident.

position in the putamen and the globus pallidus. The A3243G mutation was present in all tissues studied.

\section{DISCUSSION}

In our study, the A3243G mutation was identified in patients who presented with stroke-like episodes in addition to other classical symptoms of the MELAS syndrome (Group 2, Table 1). The mutation was not associated with ischemic stroke of undetermined origin in the absence of a typical MELAS phenotype.

Majamaa and colleagues ${ }^{19}$ found the A3243G mutation in 2/38 (6\%) of patients aged 18-45 with occipital infarcts in Finland. The two patients had clinical features of mitochondrial disorders. Martínez-Fernandez and colleagues ${ }^{6}$ suggested that mitochondrial disorders might be responsible for up to $22-33 \%$ of cryptogenic strokes in young patients but found the A3243G mutation in only 1 patient (5.6\%) who had typical symptoms and signs of MELAS among 18 young patients with cryptogenic strokes or transient ischemic attacks in the carotid or in the vertebrobasilar territories. Therefore, in agreement with our findings, the A3243G mutation has only been reported in stroke patients with typical characteristics of MELAS. Likewise, even though it has been suggested that abnormal mitochondrial function might be involved in migraine, the $A 3243 \mathrm{G}$ mutation was found neither in patients with migraine without aura and maternal history of migraine ${ }^{20}$ nor in patients with migraine with prolonged aura ${ }^{21}$.

Once MELAS is suspected, family history, measurement of lactate in blood and cerebrospinal fluid, creatine kinase, MRI, muscle biopsy and genetic testing contribute to confirm the clinical suspicion ${ }^{22}$. All patients in Group 2 showed increased lactate levels in blood and/or CSF. This finding is caused by impaired oxidative metabolism, is nonspecific but is important even though it varies in severity and may be absent in some patients.

The modified Gomory - Trichrome stain, SDH and COX reactions are crucial in diagnosis of oxidative phosphorylation diseases ${ }^{8}$. RRFs, frequently found in mitochondrial diseases ${ }^{23}$, were present in all muscle biopsies in Group 2. RRFs in Gomori-Trichrome staining (Fig 1) represent proliferation of subsarcolemmal and intermyofibrillar mitochondria with myofibril degeneration. They also have mild accumulations of glyicogen and lipid ${ }^{8}$. Most RRFs also show increased SDH reaction and decreased or absent COX reaction. In patients in Group 2, these histological changes were more frequent than ultrastructural abnormalities reported in MELAS and other mitochondrial diseases ${ }^{8}$.

The A3243G mutation was present in blood leukocytes in all patients in Group 2; in Patient 3, it was also present in different tissues in postmortem analysis, reflecting the systemic nature of the mitochondrial disorder. It is the most common mutation described in MELAS but may be present in other conditions such as Kearns-Sayre syndrome, progressive chronic external ophthalmoplegia ${ }^{24}$, pure mitochondrial myopathies and in maternally transmitted diabetes mellitus and deafness ${ }^{25}$. Conversely, cases of MELAS mutations without RRFs in muscle biopsy have been reported ${ }^{26}$.

Stroke-like episodes occur in more than $90 \%$ of patients with MELAS but their pathogenesis is controversia ${ }^{27}$. In general, symptoms in mitochondrial disorders are related to involvement of tissues with 
Table 1. Subject characteristics.

\begin{tabular}{|c|c|c|c|}
\hline & Group 1 & Group 2 & Group 3 \\
\hline Number of subjects & 14 & 3 & 20 \\
\hline Age (mean \pm standard deviation) & $34 \pm 9.4^{*}$ & $24 \pm 8.8^{*}$ & $28 \pm 6.7 *$ \\
\hline Gender (M/F) & $9 / 5$ & $2 / 1$ & $6 / 14$ \\
\hline \multicolumn{4}{|l|}{ Clinical features } \\
\hline Stroke or "stroke-like" episodes & & & - \\
\hline Anterior circulation only & 10 & 0 & \\
\hline Posterior circulation only & 3 & 0 & - \\
\hline Anterior + Posterior circulation & 1 & $3 * *$ & \\
\hline Migraine & 3 & 2 & 4 \\
\hline Recurrent vomiting & 0 & 2 & 0 \\
\hline Seizures & 0 & 3 & 0 \\
\hline Encephalopathy & 0 & 2 & 0 \\
\hline Diabetes mellitus & 1 & 1 & 0 \\
\hline \multicolumn{4}{|l|}{ Muscle biopsy } \\
\hline RRF & & $3 / 3$ & \\
\hline $\mathrm{SDH}+$ & & $3 / 3$ & \\
\hline COX- & - & $3 / 3$ & - \\
\hline mitochondria & & $2 / 3^{\#}$ & \\
\hline paracrystalline inclusions & & $0 / 3^{\#}$ & \\
\hline A3243G mutation & 0 & $3 / 3$ & 0 \\
\hline
\end{tabular}

high oxidative metabolism. Stroke-like episodes may be caused by energy failure due to mitochondrial dysfunction in neural tissue ${ }^{27}$ or by "mitochondrial angiopathy", caused by mitochondrial dysfunction in small cerebral blood vessels (ischemic hypothesis) ${ }^{28}$. Chronic lactic acidosis in MELAS may also contribute to promote further neuronal injury ${ }^{29}$.

Maps from DWI MRI images reveal decreased apparent diffusion coefficients (ADCs) due to cytotoxic edema in ischemic strokes. ADCs have been reported to be normal ${ }^{30}$ or increased ${ }^{8,22}$ in stroke-like lesions. Therefore, normal/increased ADCs in DWI sequences and normal perfusion results may help to differentiate stroke-like lesions from ischemic strokes. However, ADC decrease has also been reported ${ }^{22}$. Increased lactate in lateral ventricles, occipital and temporal lobes have been reported in patients with MELAS. Unfortunately, DWI and spectroscopy were not available at the time our patient with MELAS suffered stroke-like episodes.

This study has some limitations. First, we have not searched for other mutations that have been associated with the MELAS phenotype. Nonetheless, we have screened patients for the A3243G mt DNA mutation, reported in approximately $80 \%$ of cases of MELAS. In addition, we searched for the T3271C mtDNA mutation, described in about $10 \%$ of cases and previously reported in Brazilian patients ${ }^{11}$. Therefore, we excluded detection of important mutations by blood screening. Second, in Group 1, ischemic stroke of posterior arterial territory comprises only one third of the patients. It is possible that including patients with posterior circulation infarcts might have increased the odds of diagnosing MELAS in cryptogenic strokes. Still, in patients in Group 2, lesions did not respect strict arterial territory limits and involved brain regions from anterior and posterior arterial territories. Our purpose was not to investigate the value of screening for MELAS mutations in cryptogenic strokes in the posterior circulation territory, but in any cryptogenic strokes. Third, muscle biopsy was not performed in patients in Group 1. Therefore, biochemical analyses of enzymatic activity of mitochondrial respiratory chain complexes in muscle biopsies were not available. Heteroplasmia (the coexistence of normal and mutant mtDNA in individual tissues and high variability of mutation load in different organs) is frequent in most pathogenic mtDNA mutations, including the $A 3243 G$ mutation ${ }^{22,27}$. When the mutant mtDNA reaches a critical level, cell phenotypes change from normal to abnormal ${ }^{8}$. Mutant mtDNA levels are higher in muscle than in blood and therefore, mutations present exclusively in muscle would have been missed in patients in Group 1. However, the purpose of this study was to evaluate non-invasive screening of mi- 
tochondrial mutations associated with MELAS in cryptogenic stroke in young adults and not the sensitivity of muscle biopsy over serum analyses. Fourth, we searched for mutations in DNA isolated from blood in nonsyndromic stroke patients but a recent study has shown that sensitivity of genetic testing for mitochondrial DNA mutations is higher in nonmitotic than in mitotic tissue ${ }^{31}$. Further studies should probably include screening of mitochondrial mutations in urinary sediment and cheek mucosa. Finally, the number of patients in Group 1 was relatively small but cryptogenic strokes in young patients represent only $0.2 \%$ of all strokes In Brazil'5.

Failure in diagnosing MELAS may hinder genetic counseling, identification of asymptomatic mutation carriers and treatment of presymptomatic or earlystage disease with measures such as early diagnosis of cardiomyopathy, planning of anesthesia ${ }^{32}$ and treatment of metabolic acidosis. There is no definite clinical evidence favoring efficacy of treatment but anedoctal improvements of stroke symptoms after administration of L-arginine, coenzyme Q10 and other drugs have been reported ${ }^{22,33}$.

MELAS must be considered in patients with cryptogenic strokes with other clinical characteristics of this mitochondrial disorder. Classical criteria for this disease specified that stroke-like episodes happen before the age of 40 however, the diagnosis has been made even in the sixth decade or in the absence of family history ${ }^{22}$, as in the presented patients. A maternal pattern inheritance should be sought. Systemic symptoms are critical clues: migraine-like headaches, recurrent vomiting, encephalopathy with seizures or dementia, short stature and sensorineural hearing loss, ataxia, cardiomyopathy, diabetes mellitus, retinitis pigmentosa and proximal renal tubule defects.

Our preliminary results do not support the hypothesis that screening for the A2343G and T3271C mutations in DNA extracted from blood leukocytes are useful to diagnose oligosymptomatic forms of MELAS in these patients. We suggest that clinical findings guide mitochondrial genetic testing in stroke of undetermined origin in the young.

\section{REFERENCES}

1. Adams HP Jr., Kappelle LJ, Biller J, et al. Ischemic stroke in young adults: experience in 329 patients enrolled in the Iowa Registry of stroke in young adults. Arch Neurol 1995;52:491-495.

2. Kristensen B, Malm J, Carlberg B, et al. Epidemiology and etiology of ischemic stroke in young adults aged 18 to 44 years in northern Sweden. Stroke 1997;28:1702-1709.

3. Jacobs BS, Boden-Albala B, Lin IF, Sacco RL. Stroke in the young in the northern Manhattan stroke study. Stroke 2002;33:2789-2793.
4. Musolino R, La Spina P, Granata A, et al. Ischaemic stroke in young people: a prospective and long-term follow-up study. Cerebrovasc Dis 2003; $15: 121-128$

5. Siqueira JI Neto, Santos AC, Fabio SR, Sakamoto AC. Cerebral infarction in patients aged 15 to 40 years. Stroke 1996;27:2016-2019.

6. Martínez-Fernández E, Gil-Peralta A, García-Lozano R, et al. Mitochondrial disease and stroke. Stroke 2001;32: 2507-2510.

7. Pavlakis SG, Philips PC, Dimauro S, De Vivo DC, Rowland LP. Mitochondrial myopathy, encephalopathy, lactic acidosis, and strokelike episodes: a distinctive clinical syndrome. Ann Neurol 1984;16:481-488.

8. Shoffner JM. Mitochondrial myopathy diagnosis. Neurol Clin 2000;18:105-123.

9. Werneck LC, Abdalla H, Lohr A. MELAS (mitochondrial encephalopathy, lactic acidosis and stroke-like episodes): relato de um caso. Arq Neuropsiquiatr 1987;45:288-294.

10. Pulkes Y, Seeney MG, Hanna MG. Increased risk of stroke in patients with the A12308G polymorphism in mitochondria. Lancet 2000:356:2068-2069.

11. Iizuka T, Sakai F, Kan S, Suzuki N. Slowly progressive spread of the stroke-like lesion in MELAS. Neurology 2003:61:1238-1244.

12. Marie SKN, Goto Y, Passos-Bueno MR, et al. A Caucasian family with the 3271 mutation in mitochondrial DNA. Biochem Med Biol 1994;52:136-139.

13. Adams HP, Bendixen BH, Kappelle J, et al. Classification of subtype of acute ischemic stroke: definitions for use in a multicenter clinical trial. Stroke 1993;24:35-41.

14. Tatu L, Moulin T, Bogousslavsky J, Duvernoy H. Arterial territories of the human brain: brainstem and cerebellum. Neurology 1996;47:1125-1135.

15. Tatu L, Moulin T, Bogousslavsky J, Duvernoy H. Arterial territories of the human brain: cerebral hemispheres. Neurology 1998;50:16991708.

16. Dubowitz V. Histological and histochemical stains and reactions. In Dubowitz V, Sewry CA, Fitzsimons RB (Eds). Muscle biopsy: a practical approach. London, Bailure-Tindall; 1985:19-40.

17. The International Classification of Headache Disorders, 2.Ed. Cephalalgia 2004;24(Suppl 1):24-36.

18. Miller SA, Dykes DD, Polensky HF. A simple salting out procedure for extracting DNA from human nucleated cells. Nucleic Acids Res 1988;16:1215.

19. Majamaa K, Turkka J, Karppa M, Winqvist S, Hassinen I. The common MELAS mutation A3243G in mitochondrial DNA among young patients with an occipital brain infarct. Neurology 1997;49:1331-1334.

20. Di Gennaro G, Buzzi MG, Ciccarelli O, et al. Assessing the relative incidence of mitochondrial DNA A3243G in migraine without aura with maternal inheritance. Headache 2000;40:568-571.

21. Rozen TD, Shanske S, Otaegui D, et al. Study of mitochondrial DNA mutations in patients with migraine with prolonged aura. Headache 2004;44:674-677.

22. Dickerson BC, Holtzman D, Grant E, Tian D. Case 36-2005: a 61-year-old woman with seizure, disturbed gait, and altered mental status. NEJM 2005;353:2271-2280.

23. Sciacco M, Prelle A, Comi GP, et al. Retrospective study of a large population of patients affected with mitochondrial disorders: clinical, morphological and molecular genetic evaluation. J Neurol 2001;248:778-788.

24. Fang W, Huang CC, Lee CC, Chieng SY, Pang CY, Wei YH. Ophalmologic manifestations in MELAS syndrome. Arch Neurol 1993;50:977-980.

25. Van den Ouweland JMW, Lemkes HHPJ, Ruitenbeek LA, et al. Mutation in mitochondrial tRNA leu (UUR) gene in a large pedigree with maternally transmitted type II diabetes mellitus and deafness. Nature Genetics 1992;1:368-371.

26. Melberg A, Akerlund P, RAininko R, et al. Monozygotic twins with MELAS-like syndrome lacking ragged red fibers and lactic acidaemia. Acta Neurol Scand 1996;94:233.

27. Goto Y, Horai S, Matsuoka T, et al. Mitochondrial myopathy, encephalopathy, lactic acidosis, and stroke-like episodes (MELAS): a correlative study of the clinical features and mitochondrial DNA mutation. Neurology 1992;42:545-550.

28. Ohama E, Ohara S, Ikuta F, Tanaka K, Nishizawa M, Miyatake T. Mitochondrial angiopathy in cerebral blood vessels of mitochondrial encephalomyopathy. Acta Neuropathol 1987;74:226-233.

29. Kaufmann P, Shungu DC, Sano MC, et al. Cerebral lactic acidosis correlates with neurological impairment in MELAS. Neurology 2004;62:1297-1302.

30. Yonemura K, Hasegawa Y, Kimura K, Minematsu K, Yamagushi T. Diffusion-weighted imaging in a case of mitochondrial myopathy, encephalopathy, lactic acidosis and strokelike episodes. AJNR 2001;22:269272.

31. Shanske S, Pancrudo J, Kaufmann P, et al. Varying loads of the mitochondrial DNA A3243G mutation in different tissues: implications for diagnosis. Am J Med Genet 2004;130:134-137.

32. Thompson VA, Wahr JA. Anesthetic considerations in patients presenting with mitochondrial myopathy, encephalopathy, lactic acidosis, and strokelike episodes (MELAS) syndrome. Anesth Analg 1997;85:1404-1406.

33. Koga $Y$, Akita $Y$, Nishioka J, et al. L-arginine improves the symptoms of strokelike episodes in MELAS. Neurology 2005;64:710-712. 